\title{
MULTI-CHANNEL ADJUSTABLE DC POWER SUPPLY WITH SINGLE TRANSFORMER BASED ON SPECTRAL SEPARATION
}

\author{
Zhang Benfeng - Li Huafeng — Li Sunan *
}

\begin{abstract}
To meet the need of multi-channel DC power supply to activate multiple macro fiber composite (MFC) material simultaneously, a novel multi-channel adjustable DC supply using single-input single-output transformer based on spectral separation is proposed. A hybrid signal containing multiple frequency bands is boosted to obtain a high-voltage signal without bands change. Several frequency selection circuits are then used to separate individual signals in different frequency band from the high-voltage signal. Finally, these signals are rectified and filtered respectively to obtain multiple channel DC voltages. The feasibility of the proposed scheme is analyzed theoretically and verified by simulation. The hybrid signal containing multiple frequency bands is constructed by MCU (Micro Control Unit) and boosted using push-pull boost circuit. Low-pass, band-pass and high-pass frequency selection circuits are used to obtain the individual high-voltage signal in different frequency bands, and the amplitude frequency response characteristics of these filters are simulated using PSpice. Experimental results prove that each part of the scheme runs reliable and the output is stable and adjustable.
\end{abstract}

K e y w o r d s: spectral separation, DC supply, frequency selection, PSpice

\section{INTRODUCTION}

Piezoelectric fiber composites, also known as macro fiber composite (MFC) material, are compounded by the piezoelectric fiber and the resin matrix according to certain compositional ratio, spatial geometric distribution and connectivity method. MFC has the dual advantages of the piezoelectric ceramics and polymers by mutually gluing the rectangular piezoelectric fiber and interdigitated electrodes. Compared to traditional piezoelectric material, higher requirements on the drive signal for MFC are put forward [1-2]. MFC often requires different value DC power supply to drive, because its positive and negative withstand voltage are different. In addition, in the MFC application system, multiple MFCs are stimulated simultaneously. The demand of DC power supply will be greater.

To meet the need of a variety of DC power supplies, people tend to use several separate DC / DC power supplies to power electrical equipment. The method is simple in design, but the cost is higher and the circuit is much more complex. In addition, the bulk and weigh of power supply system are increased greatly. Therefore, multiple-output converter technology constantly attracts people's attention. Traditional multi-channel output converter topologies include: single transformer multi-winding multi-output technology, coupled inductor multiple-output technology, magnetic amplifier rear adjustment technology, synchronous switch rear adjustment technology [3]. These topologies have many shortcomings, such as: cross-regulation, mutual interference, bigger loss, narrow range of adjustment, which limit the development of multiple output DC power supply.

In this paper, a novel scheme of multi-channel adjustable DC power supply based on spectral separation using single-input single-output transformer is proposed. A hybrid signal containing multiple frequency bands is boosted to obtain a high-voltage signal without bands change. The signal in each frequency band is then extracted through the corresponding filter circuits and rectified to get multiple DC using rectifier filter finally. Each DC voltage can be adjusted separately by changing the corresponding duty cycle of the signal in each frequency band. This is the principle of multi-channel independently adjustable DC power supply using transformer.

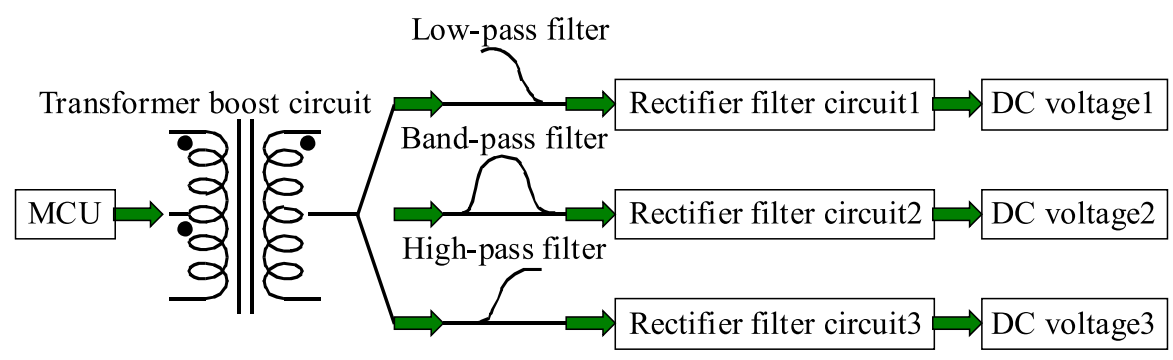

Fig. 1. Block diagram of the system

\footnotetext{
* State Key Laboratory of Mechanics and Control of Mechanical Structures, Nanjing University of Aeronautics and Astronautics, Nanjing, Box 359, No.29, Yudao Street, 210016 Nanjing, People Republic China, lihuaf@nuaa.edu.cn
} 

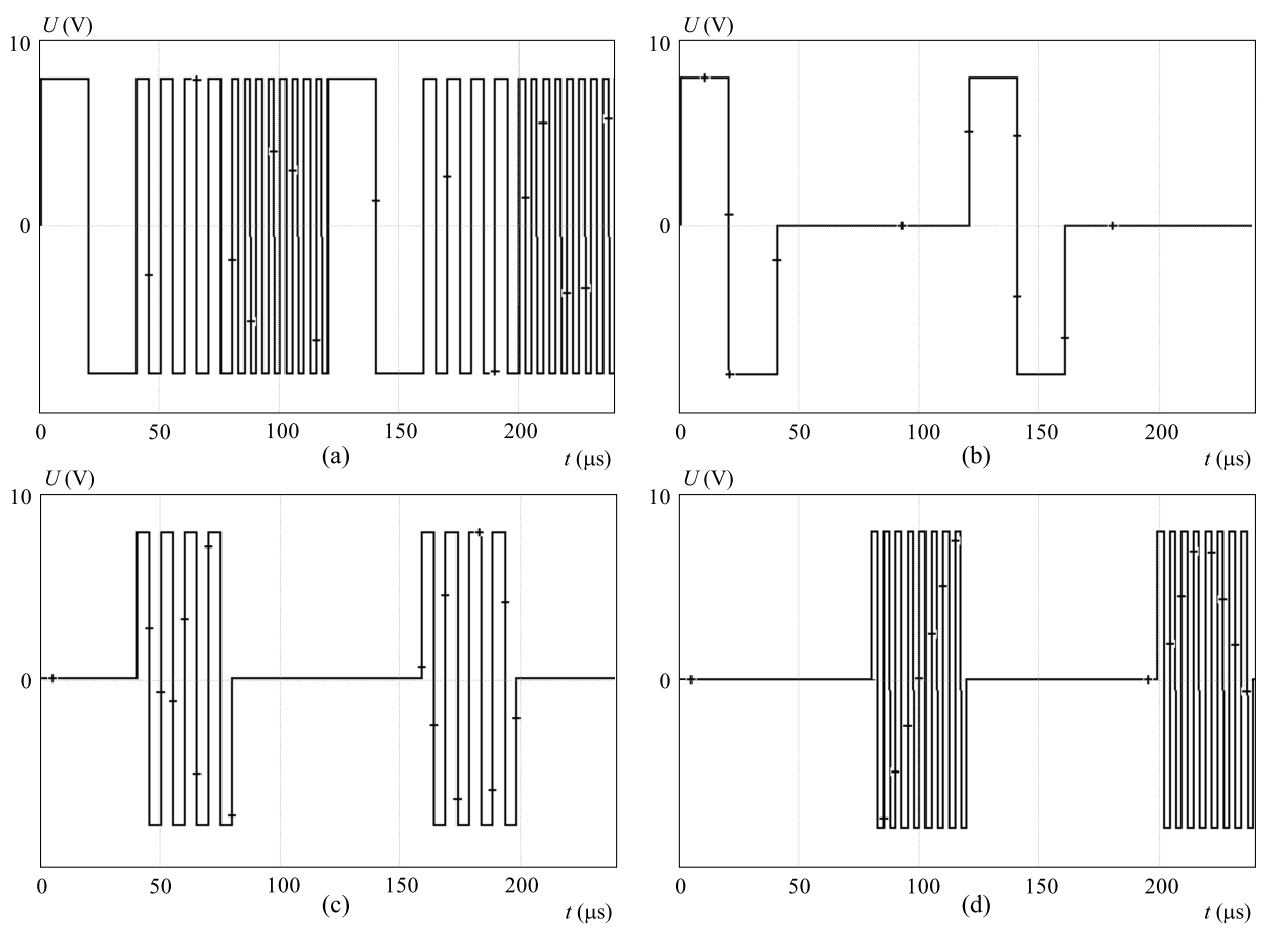

Fig. 2. The waveforms of signals $f_{a}(t), f_{b}(t), f_{c}(t)$ and $f_{d}(t)$
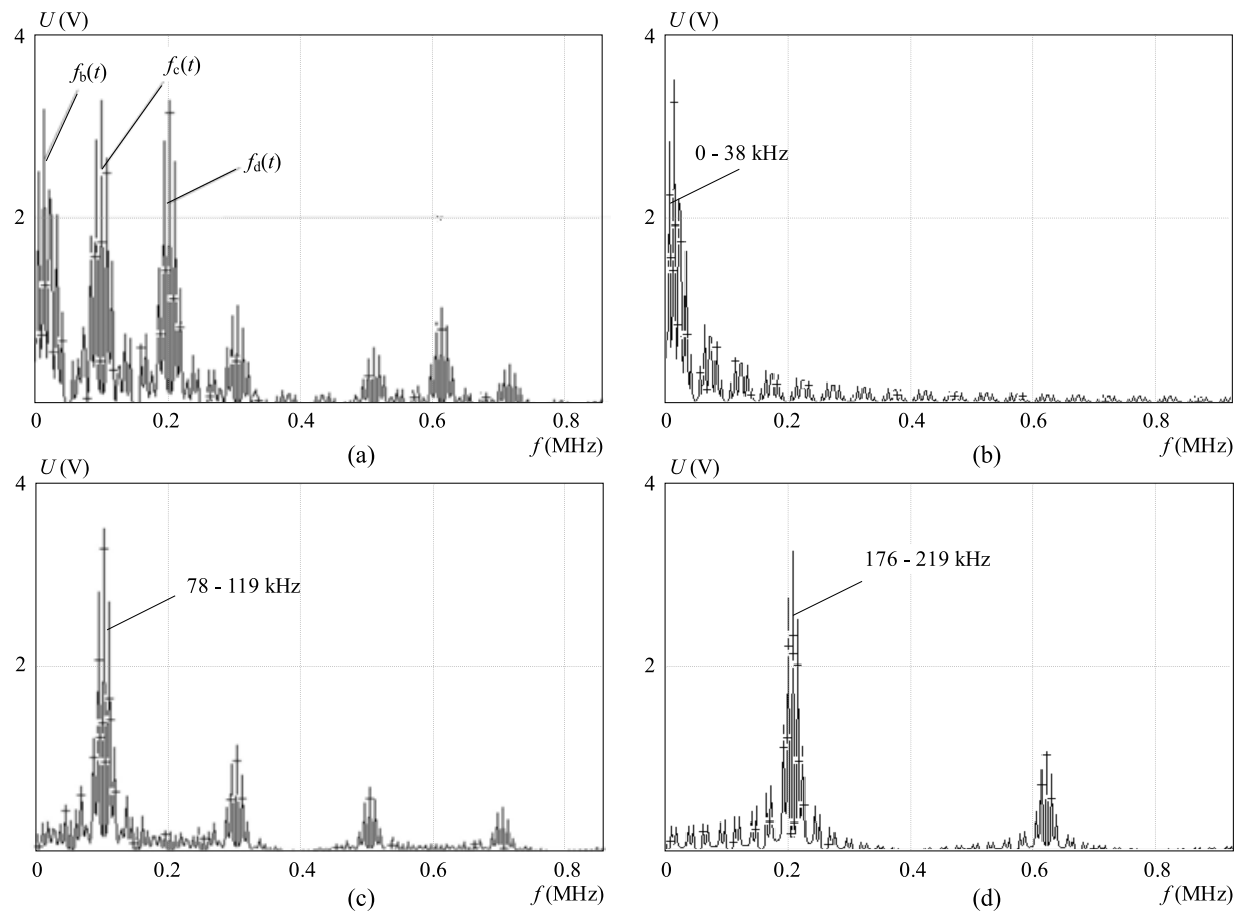

Fig. 3. The spectra of signals $f_{a}(t), f_{b}(t), f_{c}(t)$ and $f_{d}(t)$

\section{SYSTEM COSTITUTION}

According to the principle of the multi-channel DC power supply, a multi-channel DC power supply is shown in Fig. 1.

As mentioned above, this scheme is based on spectral separation technology. The third generation embedded MCU of cypress is used as signal generating and function control unit [4]. It also includes push-pull boost circuit, high-pass filter, band-pass filter, low-pass filter and three rectifier filters. In the system, MCU is used to generate a hybrid signal containing multiple frequency bands which is input push-pull boost circuit and is boosted by transformer boost circuit to get a high-voltage signal without bands change [5-6]. Then, the high-voltage signal is respectively input high-pass filter, band-pass filter and low- 

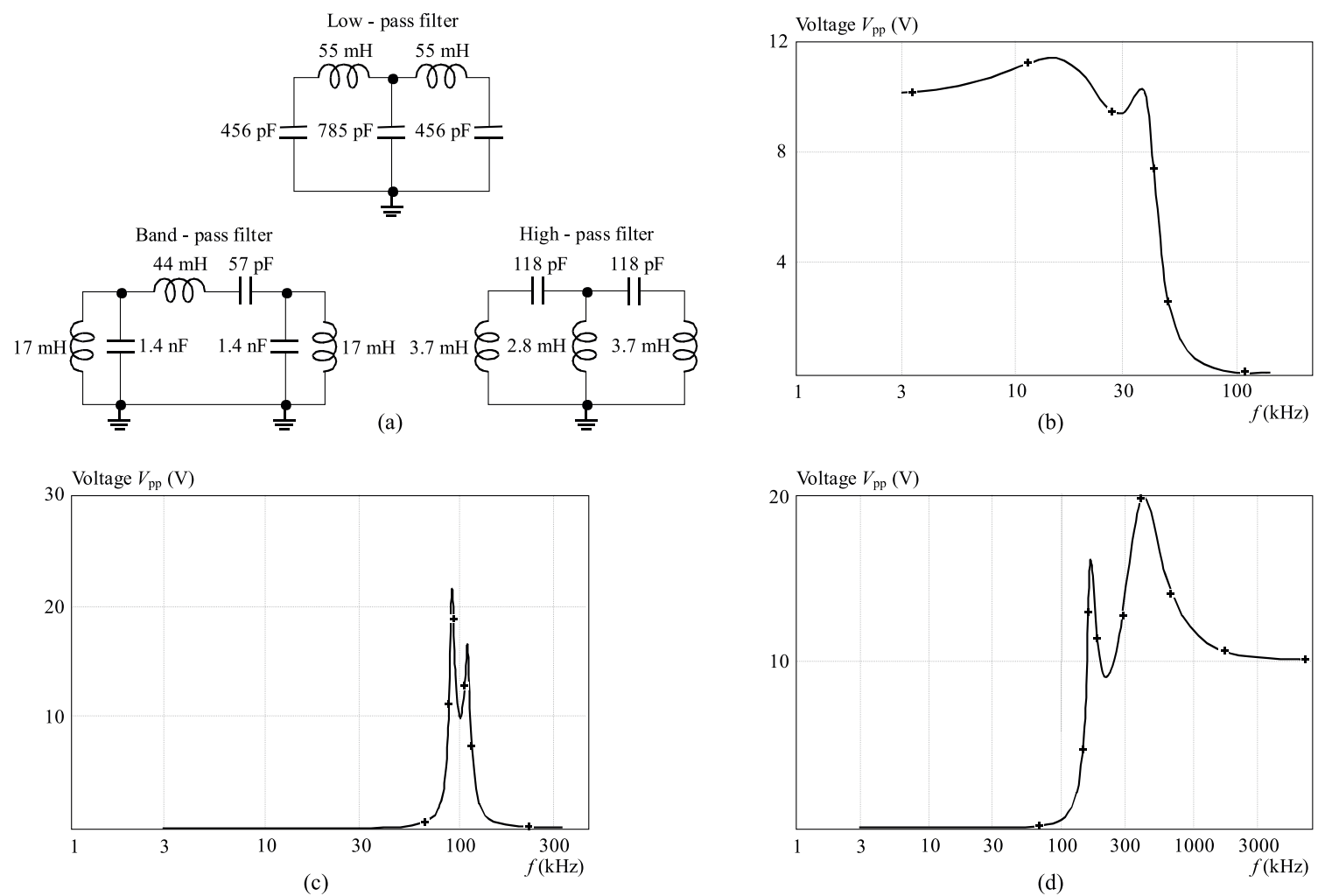

Fig. 4. The filter circuit and the frequency response curves of filters

pass filter to extract the signal in each frequency band. Finally, the signals extracted by the filters are rectified and filtered to get multiple DC using rectifier filters.

\subsection{Construction of multiple frequency bands driving signal}

The core of the multi-channel DC power supply is to construct a hybrid signal, which is a linear superposition of a few signals in several different frequency bands. Suppose signal $f_{0}(t)$ is a linear superposition

$$
f_{0}(t)=f_{1}(t)+f_{2}(t)+\cdots+f_{n}(t)
$$

Furthermore, the power spectra of $f_{1}(t)+f_{2}(t)+\cdots+f_{n}(t)$ are in different frequency bands and there is no band overlap between these signals.

$$
\text { Applying Fourier transform } F_{n}(\omega)=\int_{-\infty}^{+\infty} f_{n}(t) e^{-\mathrm{j} \omega t} \mathrm{~d} t
$$
on both sides of (1) we get

$$
F_{0}(\omega)=F_{1}(\omega)+F_{2}(\omega)+\cdots \cdots+F_{n}(\omega)
$$

In this manner the signal shown in Fig. 2 (a) is constructed, as a linear superposition of the signals shown in Fig. 2 (b), (c) and (d). Their spectra are shown in Fig. 3 (a), (b), (c) and (d). According to Fig. 3, we see that the spectrum of signals $f_{b}(t), f_{c}(t)$ and $f_{d}(t)$ are in different frequency bands. In addition, the spectrum of $f_{a}(t)$ is the linear superposition of the spectra of signals $f_{b}(t)$, $f_{c}(t)$ and $f_{d}(t)$. Therefore, theory and simulation can be verified each other, mutually.

Signal $f_{a}(t)$ is the input of a push-pull boost circuit to get a high-voltage waveform. Then, the high-voltage signal is respectively input of a high-pass filter, band-pass filter and low-pass filter to extract the signal in each frequency band. Finally, the signal extracted by filters is respectively input of a rectifier filter circuit to get multiple DC. Each DC voltage can be adjusted separately when the corresponding duty cycle of each signal is changed. Because the interference between each two bands is small, DC voltage can be changed by changing the corresponding duty cycle of signals $f_{b}(t), f_{c}(t)$ and $f_{d}(t)$ without the problem of cross-regulation.

\subsection{Boost circuit}

In order to get a high-voltage signal, the signal needs to be boosted. Linear amplification circuit is much more complex and less efficient, and push-pull boost circuit is simple, therefore it is chosen in this project [7].

In addition, the design of transformer needs more attention. Since the spectrum of signal $f_{a}(t)$ is mainly in three frequency bands, in order to ensure that the transformer is suitable for signal $f_{b}(t), f_{c}(t)$ and $f_{d}(t)$, it should be designed according to the lowest frequency band $[8]$. 


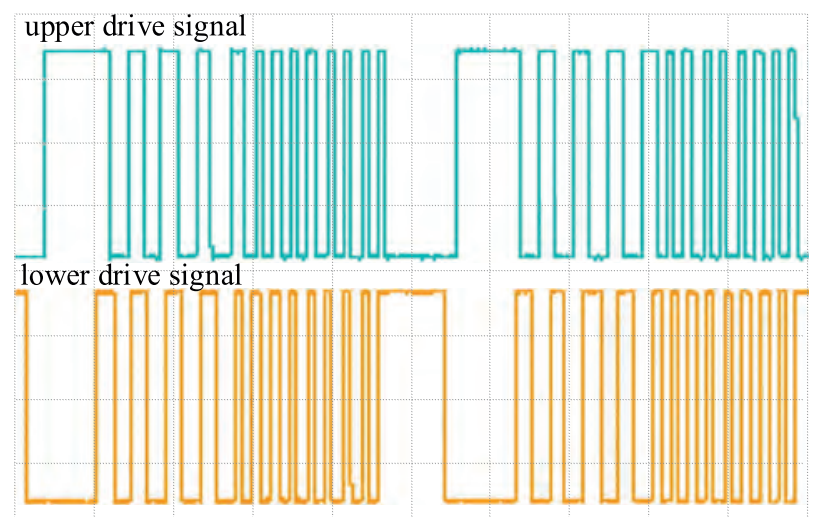

$1 \mathrm{~V} / \mathrm{div}, 25 \mu \mathrm{s} / \mathrm{div}$

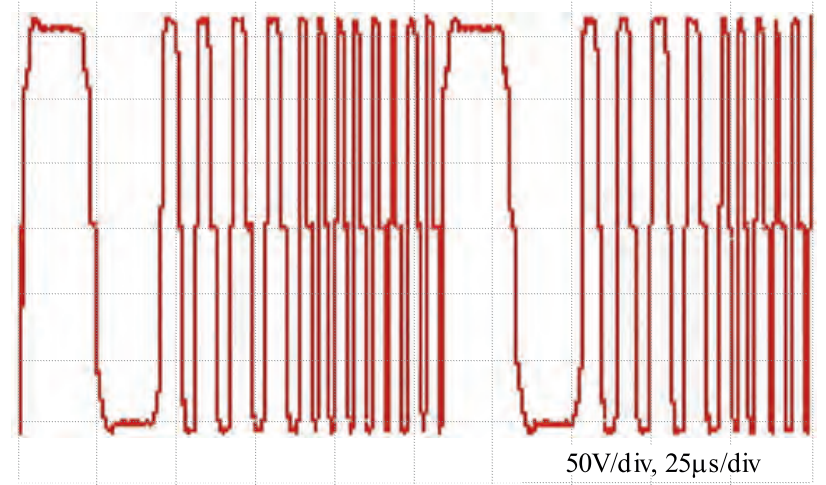

Fig. 5. The output waveform of PSoC3

Fig. 6. The output waveform of transformer

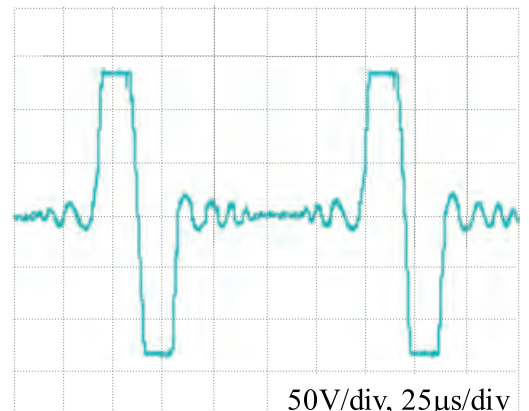

$50 \mathrm{~V} / \mathrm{div}, 25 \mu \mathrm{s} / \mathrm{div}$
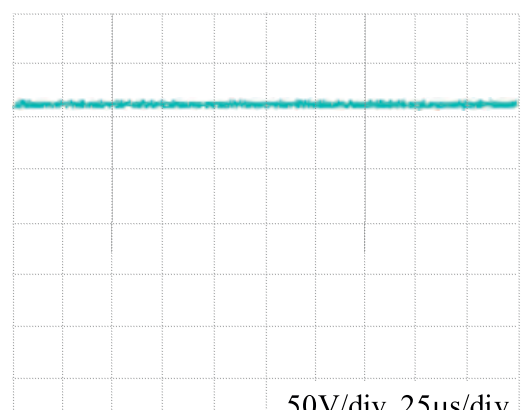

$50 \mathrm{~V} / \mathrm{div}, 25 \mu \mathrm{s} / \mathrm{div}$
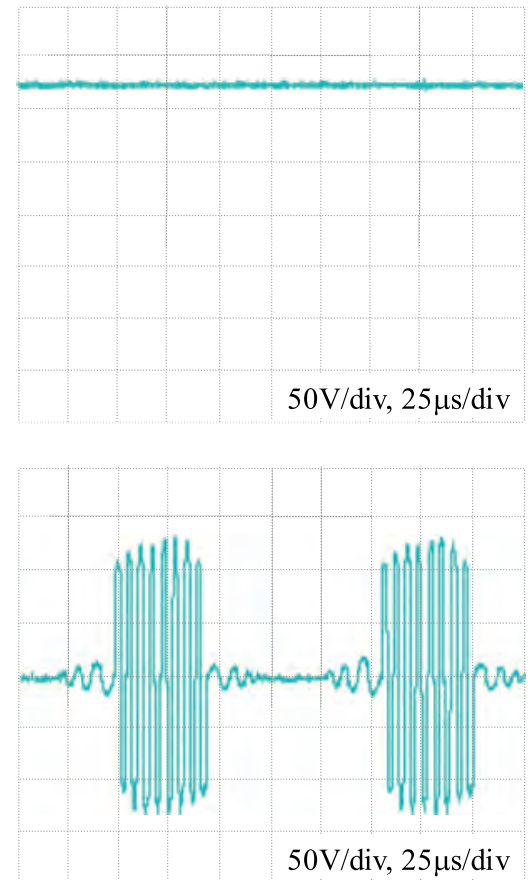
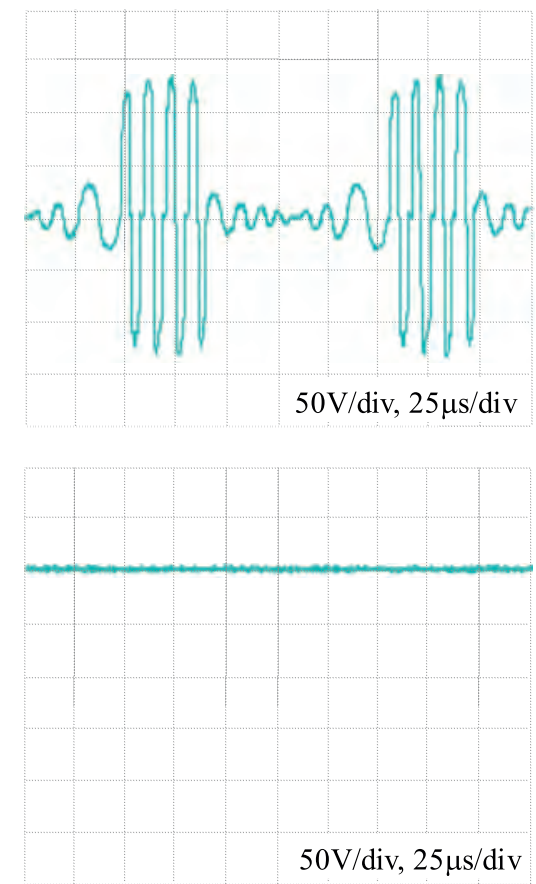

Fig. 7. The output waveforms of filters and DC waveforms for each filter

\subsection{Spectral separation circuit}

Spectral separation is a key part of the system. In the system, filters are used to achieve spectral separation. Since the filtered signal is a high voltage, $\mathrm{LC}$ passive filter is used rather than an active circuit [9].

To filter three signals, low-pass, band-pass and highpass filters were designed. The main frequency bands of signal $f_{b}(t), f_{c}(t)$ and $f_{d}(t)$ are located in $(0-38) \mathrm{kHz}$, $(78-119) \mathrm{kHz},(176-219) \mathrm{kHz}$ respectively. The interval between each two adjacent frequency bands is small. In order to get better filtering performance, 5 -th order Chebyshev filter are adopted for both high-pass and low-pass filter, and 3-rd order Chebyshev filter for band-pass fil- ter. Figure 4(a) shows the circuits of these filters. In order to examine the filtering performance of the filters, the frequency response characteristics of the filters are simulated using PSpice [10]. The input is a sine signal with $10 \mathrm{~V}$ peak to peak, the frequency response results are shown in Fig. 4 (b), (c) and (d). According to these figures, the cut-off frequency of low-pass filter is $60 \mathrm{kHz}$, the cut-off frequencies of band-pass filter are $70 \mathrm{kHz}$ and $130 \mathrm{kHz}$, and the cut-off frequency of high-pass filter is $158 \mathrm{kHz}$. Therefore, these filters have good filtering performance and meet spectral separation requirements.

The rectifier circuit used here is full-bridge circuit, and a capacitor is used to filter the signal to get DC power[11]. 


\section{EXPERIMENTAL RESULTS}

In order to prove the feasibility of the scheme, we built the entire system and made some experiments. The drive waveforms for the push-pull MOSFETS, which were generated by MCU [12-13] are shown in Fig. 5. The highvoltage signal after the amplification by transformer is shown in Fig.6. Then, the high voltage signal after passing respective low-pass, band-pass and high-pass filters are shown in Fig. 7 (a), (b) and (c). In order to obtain DC finally, these signals are input of a rectifier filter circuit to get the three DC voltages, as shown in Fig. 7 (d), (e) and (f). According to the experimental results, DC outputs of the system have small ripple and meet the requirements.

\section{CONCLUSIONS}

In this paper, a novel scheme of multi-channel adjustable DC supply with single transformer based on spectrum separation was proposed. A hybrid signal containing multiple frequency bands is boosted to obtain a highvoltage signal. The signal in each frequency band is then extracted through the filter circuits and rectified to get three DC using rectifier filter finally. The associated circuits have been built and the entire system is verified by testing. Experimental results prove that the scheme is feasible and meets the requirements of multi-channel DC power. Theory and simulation both prove the feasibility of the scheme.

\section{Acknowledgement}

The work was sponsored by the National Nature Science Foundation of China (51175250) and the Fundamental Research Funds for the Central Universities, NO. NJ20130006.

\section{REFERENCES}

[1] NAGATA, Y.-PARK, S. : Development of Underwater Robot using Macro Fiber Composite, IEEE/ASME International Con- ference on ASME Advanced Intelligent Mechatronicss (2008), 955-960.

[2] ERTURK, A.-DELPORTE, G. : Underwater thrust and power generation using flexible piezoelectric composites: an experimental investigation toward self-powered swimmer-sensor platforms, Smart Materials \& Structures (2012), 123-156.

[3] DOBBSB. G.-CHAPMANP. L.: A multiple-input DC-DC converter topology, IEEE Power Electronics Letters No. 1(1) (2003), 6-9.

[4] He Bin. Design Guide of Programmable System on Chip, Chemical Industry Press, 2011.

[5] LIY.-DONGSHENG, Y.-XINBO, R.: A new double-input DC-DC converter, Transactions China Electrotechnical Society 23 No. 6 (2008), 77-82.

[6] LEU, C. S.-HUA, G. C.-LEE, F. C.-ZHOU. C. : Analysis and design of R-C-D clamp forward converter, Proceeding of VPEC (1992), 113-120.

[7] ZHANSONG, Z.-XUANSAN, C.: Principle and Design of Switching Power Supply, Electronics Industry Press (1998).

[8] HUiZHEN, Y.-XINGZHOU, Y. : Novel Switching Power Supply, National Defence Industry Press (1996).

[9] RANDALLW. R. : HF Filter Design and computer Simulation, Noble Press, 1994.

[10] LECORBEILLER, R.: Matrix Analysis of Electric Networks, Harvard University Press, 1950.

10] CHEN, Y. M.-LIU, Y. C.-WU, F. Y.: Multi-input DC/DC converter based on the multiwinding transformer for renewable enemy applications, IEEE Transactions on Industry Applications 38 No. 4 (2002), 1096-1104.

12] ADEL, S.-SMITH, C. S.-SMITH, C. K.: Microelectronic Circuit, Oxford University Press, 1998.

[13] THOMASL. F.: Fundamentals of Analog Circuit, Prentice Hall Inc, 2000.

Received 6 January 2013

Zhang Benfeng Master, College of Aerospace Engineering at Nanjing University of Aeronautics and Astronautics, Nanjing. He was born in 1990. His research field is drive technology of piezoelectric actuator.

Li Huafeng, Professor at Nanjing University of Aeronautics and Astronautics, Nanjing, China. He was born in 1974 and obtained his Bachelors Degree and Doctors Degree of Engineering at HUST, June 1997 and June 2002 respectively. His research field is ultrasonic motor and its control system.

Li Sunan Master, College of Automation Engineering at Nanjing University of Aeronautics and Astronautics, Nanjing, China. He was born in 1989. His research field is power electronics and drives.

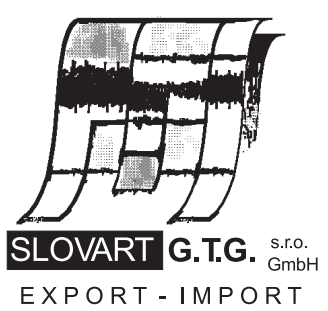

EXPORT - IMPORT
E X P OR T - I M P OR T

of periodicals and of non-periodically printed matters, books and CD-ROMs

Krupinská 4 PO BOX 152, 85299 Bratislava 5, Slovakia tel: ++421 263839 472-3, fax: ++421263839485 info@slovart-gtg.sk; http://www.slovart-gtg.sk

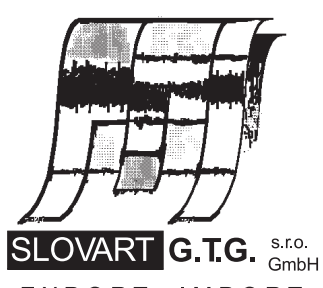

EXPORT - IMPORT 